\title{
Light Scattering from Human Hair Fibers
}

\author{
Stephen R. Marschner \\ Cornell University
}

\author{
Steve Worley \\ Worley Laboratories
}

Henrik Wann Jensen

University of California—San Diego
Mike Cammarano
Stanford University

\begin{abstract}
Light scattering from hair is normally simulated in computer graphics using Kajiya and Kay's classic phenomenological model. We have made new measurements of scattering from individual hair fibers that exhibit visually significant effects not predicted by Kajiya and Kay's model. Our measurements go beyond previous hair measurements by examining out-of-plane scattering, and together with this previous work they show a multiple specular highlight and variation in scattering with rotation about the fiber axis. We explain the sources of these effects using a model of a hair fiber as a transparent elliptical cylinder with an absorbing interior and a surface covered with tilted scales. Based on an analytical scattering function for a circular cylinder, we propose a practical shading model for hair that qualitatively matches the scattering behavior shown in the measurements. In a comparison between a photograph and rendered images, we demonstrate the new model's ability to match the appearance of real hair.
\end{abstract}

CR Categories: I.3.7 [Computer Graphics]: Three-Dimensional Graphics and Realism-Shading

Keywords: hair, fibers, optical scattering, rendering

\section{Introduction}

Realistically rendering hair is essential to portraying people and animals, but achieving realism means confronting many challenges inherent in the structure and behavior of hair. The geometry of thousands to millions of strands must be determined; the scattering of light from the fibers must be simulated; and the resulting thin curves in the image must be sampled without introducing aliasing. Most of the research effort on hair has gone toward modeling and animating the geometry of a collection of fibers [Sourin et al. 1996; Chen et al. 1999; Hadap and Magnenat-Thalmann 2000; Kim and Neumann 2000; Kim and Neumann 2002; Chang et al. 2002; Magnenat-Thalmann et al. 2002], efficient antialiasing and curve-drawing algorithms for scanline rendering [Watanabe and Suenaga 1992; Kong and Nakajima 2000], and approximating shadows within the hair [Lokovic and Veach 2000; Kim and Neumann 2001]. However, considerably less attention has been put toward the scattering model that determines the appearance of a particular assembly of fibers. Sometimes models for reflection from grooved surfaces [Kajiya 1985; Poulin and Fournier 1990] are

\author{
Pat Hanrahan \\ Stanford University
}

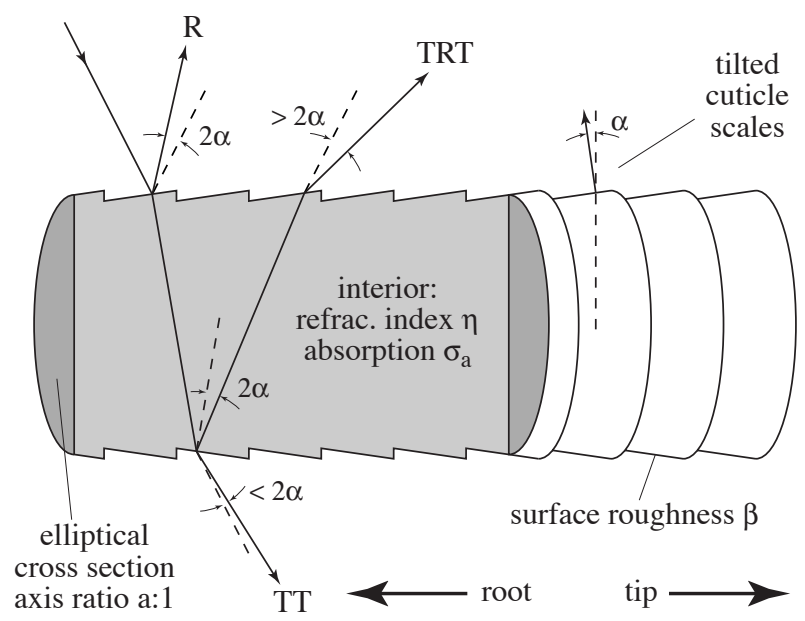

Figure 1: A schematic of our model for a hair fiber. The dashed lines indicate the scattering angles for a cylinder without tilted surface scales.

used to compute scattering from combed fibers, but most hair renderings have used the classic phenomenological model of Kajiya and Kay [1989].

Kajiya and Kay's model was designed to capture the most obvious feature of scattering from a fiber-namely the appearance of a linear highlight in the image running perpendicular to the fiber directions. It is based on the observation that the reflection of a parallel beam from the surface of a cylinder will be in a cone centered on the hair axis. The model places a constant-intensity highlight centered on that cone. All other scattering is accounted for by a diffuse term that produces radiance proportional to the cosine of the incident angle. Although it has served well for many years, this model falls short in several respects. For one thing, it is not energy conserving, which is important for physically based rendering.

But even when physical correctness is not needed, the KajiyaKay model fails to predict some observed visual effects. Since it models fibers as opaque cylinders, it does not account for transmission or internal reflection. Hair is a dielectric material, and blond, brown, red, or other light colored hair is very translucent. Goldman has simulated translucency by adding a directional parameter that controls the relative amount of forward transmission and backward reflection [Goldman 1997]. Kim has extended this model by proposing a two-term phase function [Kim 2002]. The first term models surface reflection as a cardioid, based on a ray density argument; our R component is similar but accounts for the Fresnel factor and handles oblique incidence. The second term models transmission as a forward scattering cosine lobe, supported by Monte Carlo computations of normal-incidence scattering from a transparent circular cylinder. We extend Kim's model by accounting for the Fresnel factor and volume absorption in an analytical model that also includes internal reflection, modeling the separation of highlights from different reflection modes, and approximating the effects of eccentricity. 
Two research groups working in the cosmetics industry have measured the scattering properties of hair fibers in order to understand what makes hair look pleasing and healthy. Stamm et al. [1977] measured relative scattering from a rack of individual fibers as a function of illumination and viewing angles for directions coplanar with the fiber axis (that is, in the incidence plane). They discovered two deviations from the expected behavior. First, the primary specular peak occurs at an angle several degrees away from the specular direction. They speculated that the angular shift is caused by the tilt of the scales forming the cuticle of the hair fiber. Second, there is a secondary lobe that occurs on the other side of the specular direction. Bustard and Smith [1991] have observed that the first peak preserves polarization, whereas the second peak is depolarized. Both groups also observed that the secondary highlight is not present in black hair. Bustard and Smith also report a preliminary observation of azimuthal scattering in the plane normal to the fiber in which they observed strong peaks, which they conjectured are internal reflection caustics. These experiments support the hypothesis that the secondary peak is due to internal reflection off the back side of the fiber.

We have made an experimental and theoretical study of the scattering of light from individual fibers of human hair, and developed a simple practical model for use in computer graphics. Specifically,

- In addition to the incidence-plane measurements of Stamm et al. and Bustard and Smith, we measure scattering in the plane normal to the fiber. We also report for the first time full 3D hemispherical scattering measurements. Several new appearance phenomena are visible in the 3D data. First, the primary specular highlight continues all the way around the hair, while the secondary highlight is confined to the side of the hair toward the source. Second, a pair of large out-of-plane peaks, or glints, are present, and as the incidence angle increases the peaks move closer to the incidence plane, eventually merging and disappearing.

- The scattering distribution, especially the secondary highlight, depends on the angle of rotation of the hair fiber about its axis. This is to be expected because hair fibers are not generally circular in cross section [Robbins 1994].

- To explain our observations, we propose a simple model based on the structure and composition of hair fibers (Figure 1). We approximate the hair as a transparent circular cylinder with a colored interior and a surface composed of rough, tilted scales. Using geometric optics, we derive predictions for the far-field scattered light distribution from such a cylinder. Our theory predicts that three transport modes are significant: surface reflection, transmission, and internal reflection.

- Based on the theory, we propose a shading model for human hair. We illustrate its use by producing several example renderings of brown hair. The shading model is simple and easily

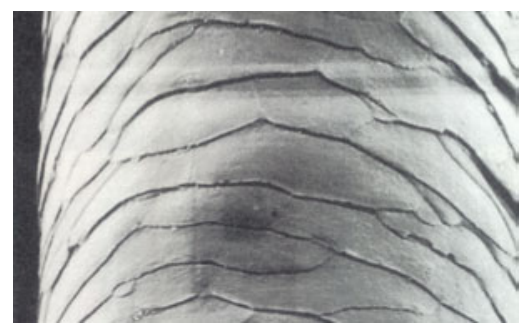

Figure 2: An electron micrograph of a hair fiber that shows the structure of the outer cuticle surface, which is composed of thin overlapping scales [Robbins 1994]. In this image the fiber is oriented with the root at the top and the tip at the bottom.

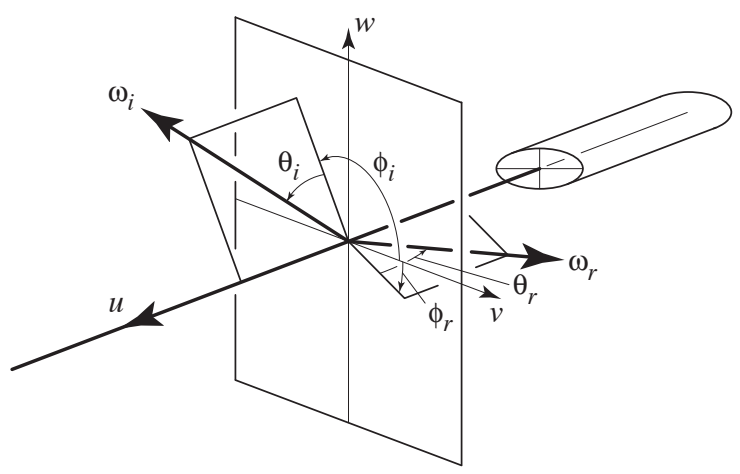

Figure 3: Notation for scattering geometry.

incorporated into standard rendering algorithms. Finally, we validate the model against experimental data and show there is good qualitative agreement.

\section{Fibers}

We begin by presenting some background information on human hair fibers. We also introduce the notation we use in this paper, and define the light scattering function for fibers.

\subsection{Hair fibers and fiber scattering}

A fiber of human scalp hair is composed of two main parts: the $\mathrm{cu}$ ticle and the cortex. The cuticle is a thin protective outside sheath that surrounds the inner cortex. The cuticle is of particular importance for light scattering, since it forms the interface between the fiber and the air. It is composed of flat cells that overlap like roof shingles (Figure 2) and make the fiber appear as a nested set of cones. Because of their overlapped arrangement, the surfaces of the scales deviate slightly but systematically from the overall normal of the fiber's surface, tilting their surfaces toward the root end of the fiber by approximately $3^{\circ}$ [Bustard and Smith 1991; Robbins 1994].

The cortex forms the bulk of the fiber. At the center is a pigmented core, the medulla. The pigments in the cortex and medulla determine the hair color. In this paper, we approximate the optical properties of the interior of the fiber with two quantities: an index of refraction $\eta$ of approximately 1.55 [Stamm et al. 1977] and an absorption cross section $\sigma_{a}$, which is assumed to be uniform throughout the fiber.

As we will see, the morphology of the hair fiber provides an explanation for the main features of the scattering function. The fiber is modeled as a dielectric cylinder covered with tilted scales and with a pigmented interior (Figure 1). Reflection of a directional beam from a cylinder produces several distinct components of reflected light, all directed into a cone of outgoing directions. Reflection from the surface produces a component that is spread fairly uniformly around the cone. Transmission through the hair produces a very bright component that is focused toward directions on the opposite side of the hair. Light that reflects off the inside of the hair surface produces a back-scattering component. This component is more complicated and, depending on the shape of the cylinder and the angle of incidence, may spread the energy over the whole cone or focus it into one or more caustics. Using $\mathrm{T}$ and $\mathrm{R}$ to stand for transmission and reflection across a cylinder interface, we denote these three modes of reflection as R, TT, and TRT.

As illustrated in Figure 1, the tilt of the cuticle scales shifts the cones of the R and TRT components slightly off the ideal specular cone. More importantly, they shift in opposite directions, causing them to separate into two visually distinguishable highlights. The 

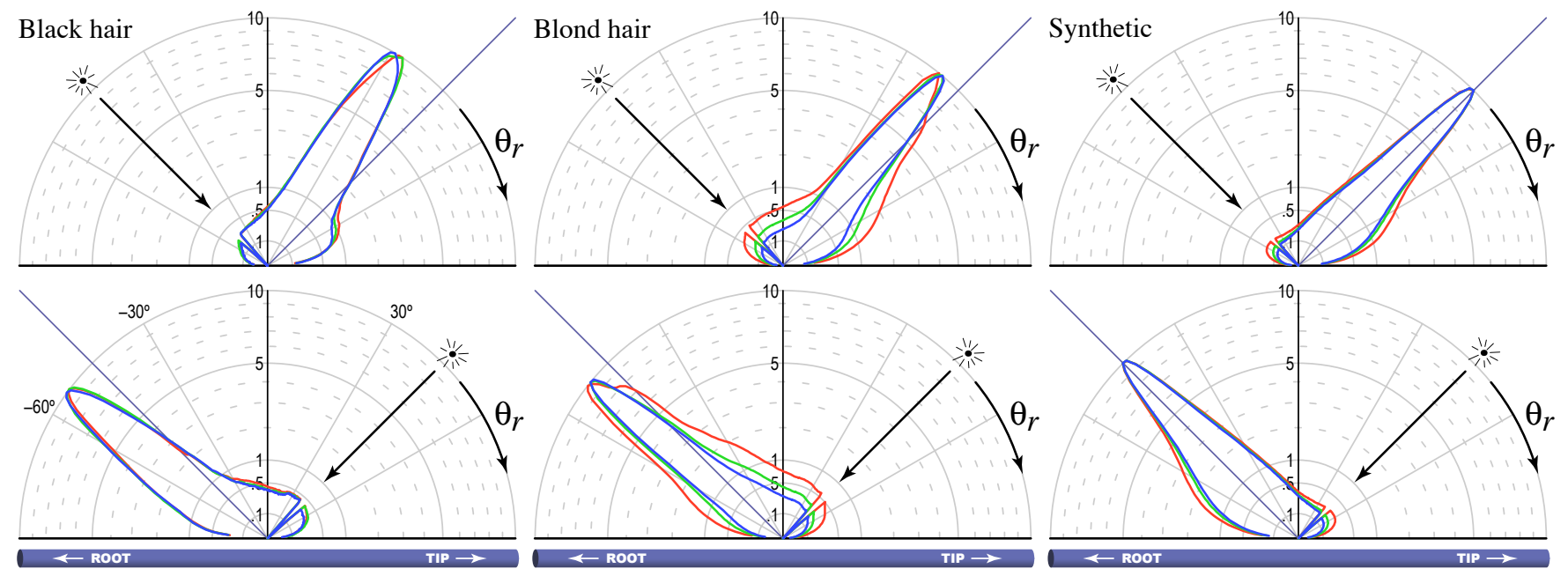

Figure 4: Measurements of scattering in the incidence plane: scattering as a function of scattering angle with illumination at $45^{\circ}$ from the tip and root ends. Black and blond hair are shown, along with a synthetic fiber from a wig.

$\mathrm{R}$ highlight, since it is surface reflection, is white, whereas the TRT highlight, which is formed by light that passes through the interior of the fiber, is colored.

\subsection{Scattering}

Before getting into more detail, let us establish the notation we will use throughout the paper to describe the scattering geometry (Figure 3). The tangent to the hair is $u$, pointing in the direction from the root toward the tip; the vectors $v$ and $w$ complete a right-handed orthonormal basis, and if the cross section is elliptical $v$ is the major axis and $w$ is the minor axis. We refer to the $v-w$ plane as the normal plane. The direction of illumination is $\omega_{i}$, and the direction in which scattered light is being computed or measured is $\omega_{r}$; both directions point away from the center. We express $\omega_{i}$ and $\omega_{r}$ in spherical coordinates. The inclinations with respect to the normal plane are denoted $\theta_{i}$ and $\theta_{r}$ (measured so that $0^{\circ}$ is perpendicular to the hair, $90^{\circ}$ is $u$, and $-90^{\circ}$ is $-u$ ). The azimuths around the hair are denoted $\phi_{i}$ and $\phi_{r}$ (measured so that $v$ is $0^{\circ}$ and $w$ is $+90^{\circ}$ ).

We also use several derived angles. The difference angle $\left(\theta_{r}-\right.$ $\left.\theta_{i}\right) / 2$ is denoted $\theta_{d}$. The relative azimuth $\phi_{r}-\phi_{i}$ is denoted simply $\phi$. The averages $\theta_{h}=\left(\theta_{i}+\theta_{r}\right) / 2$ and $\phi_{h}=\left(\phi_{i}+\phi_{r}\right) / 2$ are called half angles.

The bidirectional scattering function $S$ for a fiber is different from the bidirectional reflection distribution function $f_{r}$ for a surface, although it shares the same physical units. For the incident and reflected light we use curve irradiance $\bar{E}$, or power per unit length, and curve intensity $\bar{L}$, or intensity per unit length, respectively. These units are analogous to irradiance (power per unit area) and radiance (intensity per unit area) on a surface.

$$
S\left(\omega_{i}, \omega_{r}\right)=\frac{d \bar{L}_{r}\left(\omega_{r}\right)}{d \bar{E}_{i}\left(\omega_{i}\right)},
$$

where $\bar{L}_{r}$ is the curve intensity scattered from an infinitesimal length of fiber, and $\bar{E}_{i}$ is the curve irradiance on that portion of the fiber. This irradiance is proportional to incoming radiance:

$$
d \bar{E}_{i}\left(\omega_{i}\right)=D L_{i}\left(\omega_{i}\right) \cos \theta_{i} d \omega_{i}
$$

where $D$ is the diameter of the fiber (which depends on $\phi_{i}$ for an elliptical fiber). Note that the area over which the irradiance is measured is $D d l$ where $d l$ is an infinitesimal arc length along the fiber.
Given this definition, the scattering integral is written as

$$
\bar{L}_{r}\left(\omega_{r}\right)=D \int S\left(\omega_{i}, \omega_{r}\right) L_{i}\left(\omega_{i}\right) \cos \theta_{i} d \omega_{i}
$$

Note that, unlike a surface where the integral extends over the upper hemisphere, this integral extends over the entire sphere. The presence of $D$ in this equation indicates that a thick fiber intercepts more light, and therefore appears brighter from a distance, than a thin fiber.

\section{Scattering measurements}

The experimental component of our study of hair was intended to provide a qualitative and quantitative understanding of the phenomena that need to be explained by a scattering model for hair. In this paper, we briefly outline these measurements; a full description of our experiments will be provided in a future paper.

In our experiments we illuminated individual hairs with a narrow beam and measured the scattered light in various directions using a setup based on a four-axis goniometer that positioned a light source and a CCD camera at arbitrary directions from the sample. We used a focused beam to illuminate only a small length of hair, on the order of 1 to $2 \mathrm{~cm}$, and this illuminated segment defined the length of hair being measured. This served to reduce the effects of any variations in properties along the length of the hair.

\subsection{Incidence plane}

Stamm et al.'s and Bustard and Smith's measurements were in the incidence plane, meaning that they observed only the 2D slice of the scattering function for which the source and detector are coplanar with the fiber. To verify these earlier results, we made the same kind of measurements on samples of several different types of hair and on a synthetic fiber from a wig (Figure 4).

For each sample, we set the angle of incidence to $45^{\circ}$ and measured scattering for varying outgoing angles. We performed this experiment twice, once with the illumination from the direction of the root (with $\theta_{i}=-45^{\circ}$ ), and again with the illumination from the direction of the tip (with $\theta_{i}=45^{\circ}$ ). Each measurement was made in three color bands across the visible wavelength range. Note that the small gap near the incidence angle in each plot is due to the camera occluding the light source. 

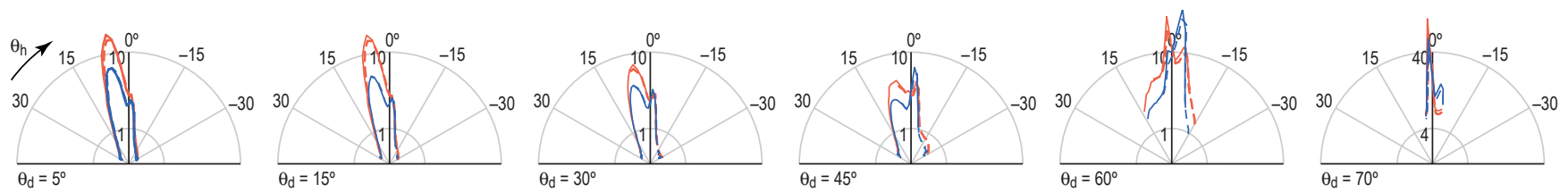

Figure 5: Measurements of scattering in the incidence plane: scattering as a function of $\theta_{h}$ for several values of $\theta_{d}$. The red and blue curves correspond to red and blue wavelength bands; for this blond hair the very strong secondary highlight can be seen to be higher in the red. The solid and dashed curves are two reciprocal measurements combined to extend the instrument's range.
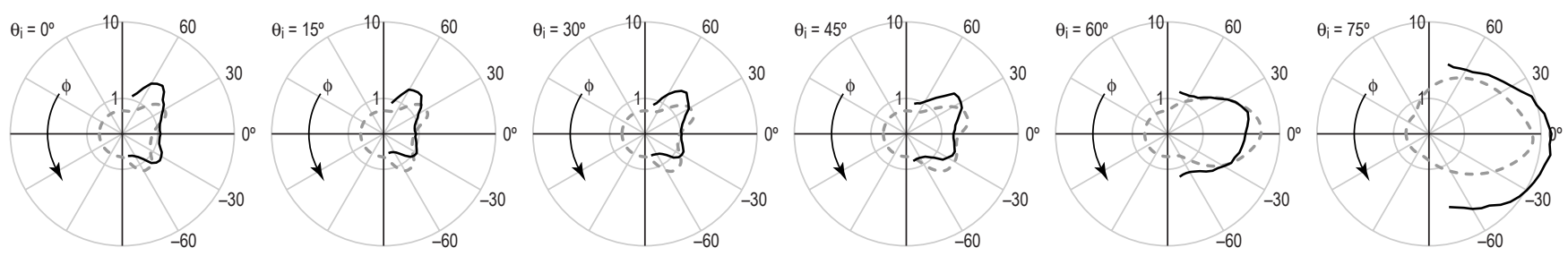

Figure 6: A measurement showing the evolution of glints with incidence angle on a more nearly circular hair. For each $\theta$ the source is fixed while the camera sweeps along the specular cone. A qualitative match to our shading model is plotted with dashed curves.

The most prominent feature in the scattering function is the specular highlight that occurs approximately when $\theta_{r}=-\theta_{i}$. For the synthetic hair the highlight occurs exactly at the specular direction, but for the real hairs it occurs 6 to 10 degrees toward the root, regardless from which direction the hair is illuminated. Additionally, the blond hair, but not the black hair, has a color-dependent asymmetry in the lobe, with a side lobe appearing closer to the specular direction than the primary highlight. A background of diffuse reflection is present that is larger and more colored for the blond hair. In other measurements of this type we always observed the secondary highlight (except in black hair), but its strength and width varied considerably.

To study the effects of both $\theta_{i}$ and $\theta_{r}$ on the scattering function in the incidence plane, we made a second measurement for one blond fiber. This measurement was parameterized in terms of $\theta_{h}$ and $\theta_{d}$. Each sweep was made with the source and detector fixed, thereby holding $\theta_{d}$ fixed, and the hair rotating to vary $\theta_{h}$.

A selection of the results of this full incidence-plane measurement are shown in Figure 5. In these plots, both the primary and secondary highlight are visible, on either side of zero, which corresponds to the specular direction. This hair in this configuration has a very strong secondary highlight that is actually stronger than the primary highlight for most angles. As the scattering angle increases, the secondary highlight fades out, while the primary highlight maintains more constant amplitude. Both peaks maintain approximately constant width. At high angles the primary highlight gives way to a sharp peak that emerges very close to the specular direction. This appears to be the same phenomenon that Stamm et al. observed and named the equal-angle peak.

\subsection{Normal plane}

To begin to understand the full 3D complexities of the scattering function, in particular the secondary highlight, we made another planar 2D measurement, but with the plane of the source and detector oriented approximately perpendicular to the fiber; that is, in the normal plane. Within this plane, the azimuths $\phi_{i}$ and $\phi_{r}$ both varied independently. To be more precise, the incident direction was in the normal plane but the scattering direction was on the cone at $\theta_{r}=10^{\circ}$. This is because the shift we have observed in the highlights due to the tilt of the cuticular scales would prevent us from observing the secondary highlight if we measured exactly in the normal plane. In this and the following measurements, we show only the red wavelength band, because that is where the secondary highlight is most prominent.

The results are plotted as solid lines in Figure 7. We have organized the data in terms of $\phi_{i}$ and the relative azimuth $\phi$, so each polar scattering diagram corresponds to a sweep of the camera with the source fixed, and moving from diagram to diagram corresponds to changing the light source angle (or equivalently, rotating the hair around its axis). The wedges of missing data are caused by the mechanical constraints of the goniometer.

This measurement shows that there are two bright out-of-plane peaks. The strength and $\phi$ locations of the peaks vary from hair to hair. We refer to this peak as a "glint" because of the visual effect it produces.

Furthermore, the glints change considerably in brightness and position as a function of $\phi_{i}$, which shows that the hair is not rotationally symmetric. However, two symmetries are evident in the figure. One is a $180^{\circ}$ rotational symmetry in $\phi_{i}$ (diametrically opposite plots are similar); the other is by negating both $\phi_{i}$ and $\phi$ (vertical bilateral symmetry of the whole figure). Together these symmetries suggest that the hair has a $180^{\circ}$ rotational symmetry and is bilaterally symmetric in cross section.

When the pattern of glints is symmetric about the plane of incidence $\left(\phi_{i}=0\right)$, it looks a lot like the caustic formation due to refraction in a sphere or cylinder with circular cross section. More generally, the evolution of the peaks as the fiber rotates appears similar to the internal reflection from a transparent elliptical cylinder, as we discuss in Section 6.1. A microscopic inspection of another fiber from the same individual reveals it has an eccentricity of $0.7: 1$.

\subsection{D hemispherical measurements}

\subsubsection{Changes in glints with angle of incidence}

It is instructive to examine the secondary highlight as a function of $\phi$ while varying the incidence angle. This shows whether and how this highlight evolves as the inclination of the hair to the light source changes. To make this measurement, we arrange for the source and camera to be at approximately equal incidence angles $\theta$ on opposite sides of the normal plane, and we measure the $\phi$ dependence by sweeping the camera along its cone while keeping the light source fixed. The results are plotted as solid lines in Figure 6.

As in the previous measurement, the camera needs to be a bit more toward the tip than the source in order to observe the maxi- 


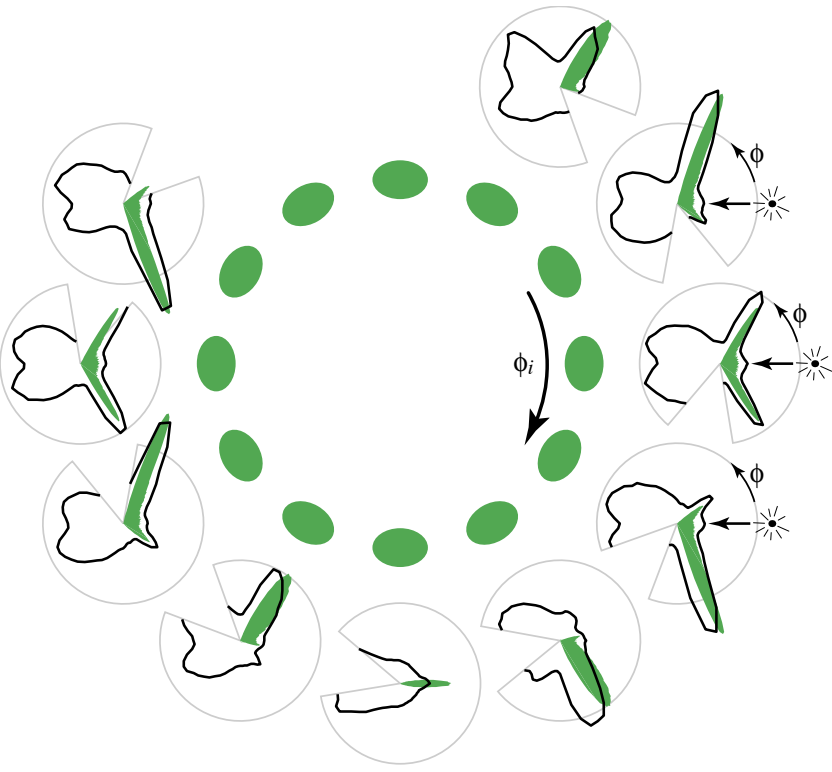

Figure 7: A measurement of scattering in the normal plane from a blond hair with substantial eccentricity. Illumination comes from the right in all plots; the green ellipses indicate the hair orientation. Bright glints appear whose location and strength depend on the orientation of the hair. The green plots are the results of Monte Carlo scattering simulation on the proposed fiber model.

mum glint intensity, so we maintain the condition that $\theta_{i}+\theta_{r}=10^{\circ}$ as we vary $\theta_{i}$.

The phenomenon shown by this measurement is that the azimuth at which the glints occur changes as a function of incidence angle, with the glints moving toward the incidence plane as the incidence moves from normal to grazing. They appear to merge around $\theta=60^{\circ}$. This behavior provides another clue to the structure: such a merging transition is characteristic of the caustics formed by internal reflection in a cylinder [Mount et al. 1998]. The measured eccentricity of this fiber is approximately $0.8: 1$.

\subsubsection{Hemispherical scattering}

These measurements extended the 1D incidence plane measurements by varying $\phi_{r}$ as well as $\theta_{r}$, so that the scattering direction varied over a full hemisphere. For a fixed direction of incidence, this is a complete measurement of the scattering function (or half of it, which suffices given bilateral symmetry-a reasonable if inexact assumption when we are not allowing $\phi_{i}$ to vary).

The results are shown in Figure 8. In this plot, several curves for fixed values of $\phi$ are plotted together against $\theta_{r}$. A primary highlight appears at $\theta_{r}=40^{\circ}$, and a broad secondary highlight appears around $50^{\circ}-80^{\circ}$. Moving from curve to curve, the primary highlight maintains a fairly constant amplitude and shift from the specular cone (marked by a blue line in the plot). The secondary highlight becomes stronger, peaks around $\phi=30^{\circ}$, and then dies out. The forward-scattering half of the scattering function, shown with a larger scale on the lower axes, is dominated by a strong forward-scattered component that is not shifted appreciably from the specular cone.

\subsection{Summary}

To summarize, our experiments measuring the scattering of light from hair fibers revealed the following phenomena. Here, we categorize the phenomena in terms of the scattering modes of a dielectric cylinder (Section 2).

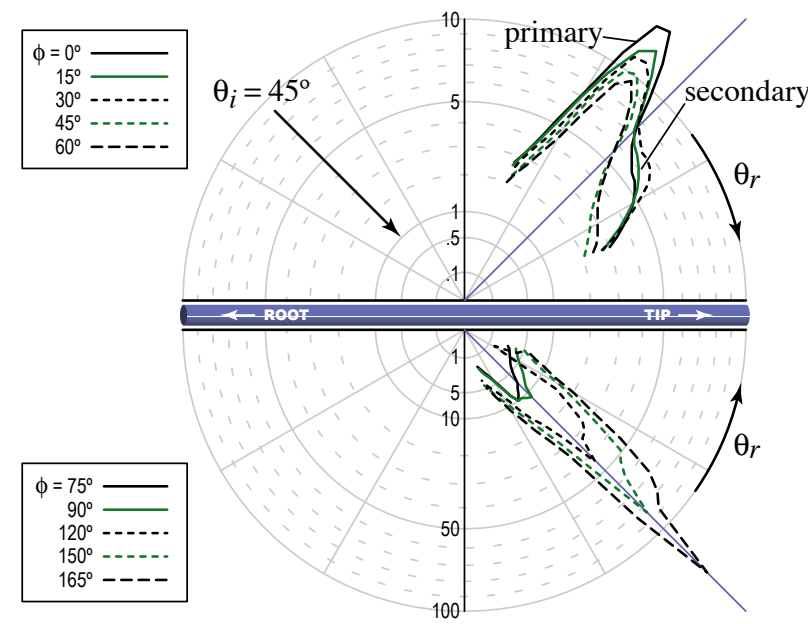

Figure 8: A measurement of one hemisphere of the scattered distribution for a fixed incident direction with angle of incidence $45^{\circ}$. The primary highlight, the secondary highlight, and the transmitted component are all visible.

- R: Shift of the primary specular peak toward the root. This deviation is hypothesized to be due to the tilt in the scales on the hair fiber.

- TT: A strong forward scattering component from light colored hair. This causes blond, brown, gray, and white hair to look very bright when lit from behind.

- TRT:

- A colored secondary peak shifted toward the tip from the white primary specular peak. In a head of hair, this leads to the secondary highlight that is visible just above the primary, sometimes appearing more as a colored fringe on the primary than a separate feature.

- The secondary highlight varies as a function of $\phi$. It generally contains two peaks, or glints. The locations of the peaks depend on the angle of incidence, and they converge to the incidence plane as $\theta$ increases. Furthermore, for hair that is not circular in cross section, the strength and position of the glints depend strongly on the angle of rotation of the hair around its axis. This gives the hair fiber a distinctive sparkling appearance.

- Most of the energy in the secondary highlight is contained between the two glints.

\section{Theory of scattering from fibers}

In this section we present background theory applicable to scattering from smooth cylinders. We show that because of the symmetry of a cylinder, the 4D scattering function can be factored into a product of two $2 \mathrm{D}$ terms. One term, called $M$, captures the $\theta$ dependence, and the other, called $N$, captures the $\phi$ dependence. We also derive $N$ for the case of a circular cylinder. These two parts form the basis for the complete, practical model developed in the next section, in which we introduce expressions for $M$, an approximation to extend $N$ to elliptical cylinders, and procedures for efficiently approximating $N$.

\subsection{Scattering from cylinders}

For fibers formed by translating a fixed cross section along a single axis we can separate the $4 \mathrm{D}$ scattering function to a product of $2 \mathrm{D}$ 
terms. We make use of properties that have been used in previous work on scattering from fibers [Marcuse 1974; Adler et al. 1998; Mount et al. 1998]:

- A ray that enters a dielectric cylinder at a particular inclination to the axis will always exit at the same inclination, regardless of the sequence of refractions and reflections it undergoes.

This means that a bundle of parallel incident rays coming from the direction $\omega_{i}$ will produce a collection of scattered rays whose directions lie on the cone centered on the fiber axis and containing $-\omega_{i}$ (Appendix A). The directions of the refracted rays inside the hair also lie on a cone. This effectively reduces the scattering function from $4 \mathrm{D}$ to $3 \mathrm{D}$, because scattering only occurs when $\theta_{r}=-\theta_{i}$. Of course, this equality holds only approximately if the surface is rough, and it depends on the assumption that there is no volumetric scattering inside the cylinder.

- The dependence of the scattered distribution on $\phi_{r}$ can be analyzed by examining only the projection into a plane perpendicular to the hair.

It's easy to see that the $\mathrm{R}$ component can be computed just from the projection - the incident and reflected vectors are arranged symmetrically across the surface normal in the projection just as they are in $3 \mathrm{D}$, so a mirror reflection from the $3 \mathrm{D}$ cylinder remains a mirror reflection in the projection.

The same holds for refracted rays, as a consequence of Bravais's law (derived in Appendix B), which states that if the incident and transmitted vectors at a dielectric interface are projected into a plane containing the surface normal, the projected vectors still obey Snell's law, but with the index of refraction $\eta$ replaced by $\eta^{\prime}(\eta, \theta)>\eta$. Note that $\eta^{\prime}$ depends only on the inclination $\theta$ of the incident ray out of the projection plane, and it is independent of the angles in the projection plane. Thus, since we know that all rays originating from a particular incident direction maintain the same inclination to the normal plane, a 2D analysis that works in the normal plane for arbitrary refractive index suffices to describe the 3D scattering function.

These two facts allow us to write the scattering function for a smooth cylinder of any cross section as:

$$
S\left(\phi_{i}, \theta_{i} ; \phi_{r}, \theta_{r}\right)=\delta\left(\theta_{r}+\theta_{i}\right) N\left(\eta^{\prime}(\theta) ; \phi_{i}, \phi_{r}\right) / \cos ^{2} \theta
$$

The first factor says that scattering only occurs in the specular cone; the second factor $N$ represents the azimuthal scattering function; and $\cos ^{2} \theta$ accounts for the projected solid angle of the specular cone. We write simply $\theta$ to emphasize that $\theta_{i}=\theta_{r}$. Since $\theta$ influences $N$ only indirectly through $\eta^{\prime}$, we can derive $N$ independently using a $2 \mathrm{D}$ analysis. In the next section, we will generalize this expression to

$$
S\left(\phi_{i}, \theta_{i} ; \phi_{r}, \theta_{r}\right)=M\left(\theta_{i}, \theta_{r}\right) N\left(\eta^{\prime}\left(\theta_{d}\right) ; \phi_{i}, \phi_{r}\right) / \cos ^{2} \theta_{d} ;
$$

that is, we will allow for a more complicated dependence on $\theta$. We will refer to $M$ as the longitudinal scattering function.

\subsection{Scattering from a circular cross section}

In this section we derive $N$ assuming a circular cross section. With circular symmetry, $N$ depends only on $\phi$, the difference between $\phi$ and $\phi_{r}$, so the 2D azimuthal scattering function is further reduced to $1 \mathrm{D}$, and in this context we will write $N$ with a single angular argument. Scattering from a dielectric circle is well studied, having been

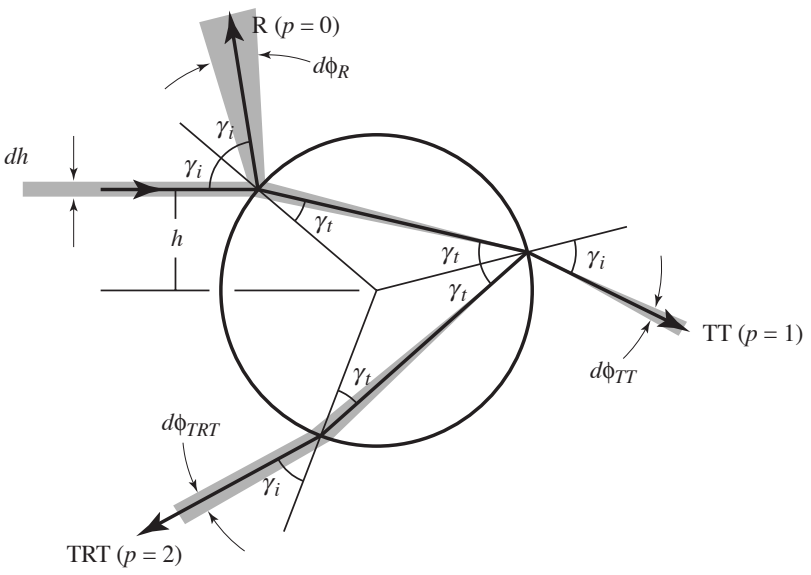

Figure 9: Geometry for scattering from a circular cross section.

first analyzed by Descartes to explain the formation of the rainbow in a rain shower (for a full discussion, see, e.g., Humphreys [1964]). Note that the effective refractive index $\eta^{\prime}$ is a parameter to the entire discussion that follows, so for clarity it is left as an implicit parameter when it is not being discussed.

We can determine the far-field intensity by tracing rays as they refract through a circle. Consider a ray incident on a unit circle at an offset $-1<h<1$ from its center (see Figure 9). The angle $\sin \gamma_{i}=h$ is the angle of incidence, and $\eta^{\prime} \sin \gamma_{t}=h$ is the angle of the refracted ray. By following a ray entering a circle as it refracts and reflects, we can calculate the exit angle $\phi(h)$.

From Figure 9 it can be seen that the incident ray deviates by $\gamma_{t}-\gamma_{i}$ as it enters the circle, by $\pi+2 \gamma_{t}$ at each internal reflection, and by $\gamma_{t}-\gamma_{i}$ again as it exits, for a total of

$$
\phi(p, h)=2 p \gamma_{t}-2 \gamma_{i}+p \pi
$$

where $p$ is the number of internal path segments. ${ }^{1}$ This expression encompasses all three scattering modes we are interested in, for different values of $p$ : surface reflection $\mathrm{R}(p=0)$, refractive transmission TT $(p=1)$, and internal reflection TRT $(p=2)$. For the rest of this analysis, we will ignore $p>2$ terms.

The above ray tracing calculation parameterizes paths by $h$. However, for scattering calculations we often need to find all the paths that contribute to scattering in a given direction $\phi$. The $h$ values for these paths are found by solving for the roots of the function $\phi(p, h)-\phi=0$. We denote these roots by the function $h(p, r, \phi)$, where different values of $r$ denote different roots. In the $p=0$ and $p=1$ cases, there is a single root, and thus a single path. However, for the $p=2$ case, there may be one or three roots and hence one or three paths.

Since the function $\phi$ is smooth, the transition from a single exit ray to three exit rays represents a fold in $\phi(2, h)$ and this fold occurs when $\frac{d \phi}{d h}=0$. Descartes first showed that this fold occurs when:

$$
h^{2}=\left(4-\left(\eta^{\prime}\right)^{2}\right) / 3
$$

This expression is symmetric, and predicts that two symmetric extrema exist. [Humphreys 1964]

Now that we have the expression (3) to relate $\phi$ and $h$, we can compute the intensity of the scattered light by using the principle of energy conservation. When curve irradiance $\bar{E}$ illuminates a fiber, uniform irradiance $E(h)=\bar{E} / 2$ falls on the cross section across its

\footnotetext{
${ }^{1}$ Note that $p \pi$ accounts for $p-1$ internal reflections plus the reversal in direction from $\omega_{i}$, which points outward.
} 
width. $^{2}$ Setting aside attenuation for the moment, power from a small interval $d h$ in the incident beam is scattered into an angular interval $d \phi$ in the exitant intensity distribution (Figure 9).

$$
\bar{L}(\phi(h)) d \phi=E(h) d h=(\bar{E} / 2) d h
$$

or

$$
\bar{L}(\phi(h))=\left|2 \frac{d \phi}{d h}\right|^{-1} \bar{E}
$$

This equation implies that intensity goes to infinity at the fold in the function $\phi$. This intensity singularity is called a caustic.

In the case of a circular cylinder, the pair of caustics cause the glints. Since in the cylinder the Bravais index $\eta^{\prime}$ increases with $\theta$, the two caustics will move closer to the plane as the incident light becomes more oblique to the hair. When $\eta^{\prime}$ reaches 2, the caustic ceases to form. This caustic merge transition can be seen in the measurement plotted in Figure 6.

\subsection{Attenuation by absorption and reflection}

In this section we add terms for volume absorption in the fiber interior and Fresnel reflection at the interfaces.

For absorption we need to know the path length inside the fiber. Applying the law of cosines to the triangles in Figure 9 gives the length of each internal path segment as $2+2 \cos \left(2 \gamma_{t}\right)$ times the hair's radius. Let $\sigma_{a}$ be the volume absorption per unit length, with the unit length defined to be the radius of the hair. Then each of the $p$ segments inside the hair contributes a factor of $T\left(\sigma_{a}, h\right)=\exp \left(-2 \sigma_{a}\left(1+\cos \left(2 \gamma_{t}\right)\right)\right)$ to the absorption.

Now we can introduce an attenuation factor in front of the intensity contributed by a path

$$
\bar{L}(\phi)=A(p, h)\left|2 \frac{d \phi}{d h}\right|^{-1} \bar{E}
$$

where

$$
\begin{aligned}
& A(0, h)=F\left(\eta, \gamma_{i}\right) \\
& A(p, h)=\left(1-F\left(\eta, \gamma_{i}\right)\right)^{2} F\left(1 / \eta, \gamma_{t}\right)^{p-1} T\left(\sigma_{a}, h\right)^{p} .
\end{aligned}
$$

Just as Bravais's law lets the normal-incidence geometry factor work for oblique incidence, we can generalize $A$ to oblique incidence simply by changing the parameters. Appendix B shows that the correct Fresnel factors may be computed using the usual Fresnel formulas and two virtual indices of refraction, $\eta^{\prime}(\theta)$ and $\eta^{\prime \prime}(\theta)$. Similarly, because all the internal path segments have the same inclination $\theta_{t}$ to the axis, they lengthen by a factor of $1 / \cos \theta_{t}$, so substituting $\sigma_{a}^{\prime}(\theta)=\sigma_{a} / \cos \theta_{t}$ for $\sigma_{a}$ completes the generalization to 3D:

$$
\begin{aligned}
& A(0, h)=F\left(\eta^{\prime}, \eta^{\prime \prime}, \gamma_{i}\right) \\
& A(p, h)=\left(1-F\left(\eta^{\prime}, \eta^{\prime \prime}, \gamma_{i}\right)\right)^{2} F\left(\frac{1}{\eta^{\prime}}, \frac{1}{\eta^{\prime \prime}}, \gamma_{t}\right)^{p-1} T\left(\sigma_{a}^{\prime}, h\right)^{p}
\end{aligned}
$$

The complete normal-plane scattering function is thus

$$
\begin{aligned}
N(\phi) & =\sum_{p} N_{p}(p, \phi) \\
N_{p}(p, \phi) & =\sum_{r} A(p, h(p, r, \phi))\left|2 \frac{d \phi}{d h}(p, h(p, r, \phi))\right|^{-1}
\end{aligned}
$$

where the sum is over all the paths of different types $(p)$ including those with multiple roots $(r)$.

\footnotetext{
${ }^{2}$ For the unit radius circle $\bar{E}$ is spread out across the fiber width of 2 . The fiber diameter does not affect $\mathrm{S}$.
}

\subsection{Summary}

The theory developed in this section predicts that the light from a directional beam will be scattered into a perfect cone and that the distribution around the cone is a sum of three distinct scattering modes. This leads to the following form for our new scattering model:

$$
\begin{aligned}
S\left(\phi_{i}, \theta_{i} ; \phi_{r}, \theta_{r}\right)= & \\
& M_{R}\left(\theta_{h}\right) N_{R}\left(\eta^{\prime}\left(\eta, \theta_{d}\right) ; \phi\right) / \cos ^{2} \theta_{d}+ \\
& M_{T T}\left(\theta_{h}\right) N_{T T}\left(\eta^{\prime}\left(\eta, \theta_{d}\right) ; \phi\right) / \cos ^{2} \theta_{d}+ \\
& M_{T R T}\left(\theta_{h}\right) N_{T R T}\left(\eta^{\prime}\left(\eta^{*}\left(\phi_{h}\right), \theta_{d}\right) ; \phi\right) / \cos ^{2} \theta_{d} .
\end{aligned}
$$

This equation derives from Equation 2 but includes generalizations that will allow us to introduce several useful approximations in the next section. The three terms $M_{R}, M_{T T}$, and $M_{T R T}$ are three separate longitudinal scattering functions. They allow the effects of the cuticle scales, which are different for the three modes, to be simulated. The three terms $N_{R}, N_{T T}$, and $N_{T R T}$ are the three modes of the azimuthal scattering function. The first two of these,

$$
\begin{aligned}
N_{R}(\phi) & =N_{p}(0, \phi) \\
N_{T T}(\phi) & =N_{p}(1, \phi),
\end{aligned}
$$

are defined directly from Equation 8, but the computation of $N_{T R T}$ will be discussed in more detail in Section 5.2.2. The function $\eta^{*}$ lets the effective index of refraction for $N_{T R T}$ depend on $\phi_{h}$, which allows the approximation for elliptical cross sections presented in Section 5.2.3.

Note that only the angles $\theta_{d}, \phi, \theta_{h}$, and $\phi_{h}$ appear on the right hand side of Equation 9. Writing the $M \mathrm{~s}$ and $N \mathrm{~s}$ in terms of sums and differences in this way emphasizes the natural symmetry of $S$ and will make it easy to see that the model is reciprocal.

\section{A practical shading model for human hair}

In this section we extend the results of the previous section into a complete, practical model suitable for integration into a renderer. To do this we introduce empirical functions for the longitudinal scattering functions $\left(M_{R}\right.$, etc.), which the theory of smooth cylinders does not predict. We also describe our approach to computing $N_{T R T}$ in the presence of surface roughness and an approximation to $h(p, r, \phi)$ that is used to compute $N_{R}, N_{T T}$, and $N_{T R T}$. Finally, we introduce convenient parameters for controlling the model.

It is important to recognize that the purpose of the model given in this section is to capture the phenomena that are important in rendering. The form of the model and the basic energy distribution come from the theory presented in the previous section, but there are many details for which a simple analysis is not available, and we fill these in empirically.

\subsection{The longitudinal scattering function $M$}

The analysis of a smooth cylinder predicts that the reflected light will stay exactly in the specular cone. Our hair model includes two features that cause deviations from this behavior. First, the interfaces are rough. As rays propagate through the cylinder, the roughness will cause their directions to deviate randomly from the directions predicted by the model. The overall effect is to blur the scattered distribution, with the different scattering modes blurred to different degrees. Second, the cuticle scales cause a tilt of the surface normals relative to the ideal cylinder, which causes the scattered lobes not to be centered on the specular cone.

Figure 1 illustrates how we can expect the positions of the lobes to shift: the R mode is displaced by $2 \alpha$ toward the root; the TRT 


\begin{tabular}{clc} 
Parameter & Purpose & Typical values \\
\hline $\begin{array}{c}\text { Fiber properties } \\
\eta\end{array}$ & index of refraction & 1.55 \\
$\sigma_{a}$ & absorption coefficient (R, G, B) & 0.2 to $\infty$ \\
$a$ & eccentricity & 0.85 to 1 \\
Surface properties & \\
$\alpha_{R}$ & longitudinal shift: R lobe & $-10^{\circ}$ to $-5^{\circ}$ \\
$\alpha_{T T}$ & longitudinal shift: TT lobe & $-\alpha_{R} / 2$ \\
$\alpha_{T R T}$ & longitudinal shift: TRT lobe & $-3 \alpha_{R} / 2$ \\
$\beta_{R}$ & longitudinal width (stdev.): R lobe & $5^{\circ}$ to $10^{\circ}$ \\
$\beta_{T T}$ & longitudinal width (stdev.): TT lobe & $\beta_{R} / 2$ \\
$\beta_{T R T}$ & longitudinal width (stdev.): TRT lobe & $2 \beta_{R}$ \\
$G l i n t s$ & & \\
$k_{G}$ & glint scale factor & 0.5 to 5 \\
$w_{c}$ & azimuthal width of caustic & $10^{\circ}$ to $25^{\circ}$ \\
$\Delta \eta^{\prime}$ & fade range for caustic merge & 0.2 to 0.4 \\
$\Delta h_{M}$ & caustic intensity limit & 0.5
\end{tabular}

Table 1: All the parameters of the shading model.

mode is displaced more than that far toward the tip; and the TT mode is displaced toward the tip. ${ }^{3}$ We approximate the effects of the scales by shifting the mean of $M$ differently for each lobe.

$$
\begin{aligned}
M_{R}\left(\theta_{h}\right) & =g\left(\beta_{R} ; \theta_{h}-\alpha_{R}\right) \\
M_{T T}\left(\theta_{h}\right) & =g\left(\beta_{T T} ; \theta_{h}-\alpha_{T T}\right) \\
M_{T R T}\left(\theta_{h}\right) & =g\left(\beta_{T R T} ; \theta_{h}-\alpha_{T R T}\right)
\end{aligned}
$$

where $g(\beta, x)$ is a unit-integral, zero-mean lobe function with width $\beta$. In our implementation we used a normalized Gaussian function with standard deviation $\beta$.

\subsection{The azimuthal scattering function $N$}

In this section we introduce three approximations to the azimuthal scattering function. The first is an efficient method for solving for paths, the second is a phenomenological model for the secondary highlights, and the third is a simple model for glints.

\subsubsection{Solving for paths}

In order to find a path through a circle, we must solve Equation 3 for the value or values of $h(p, r, \phi)$ that result in the scattering angle $\phi$. Because the formula for $\phi$ involves Snell's Law and hence arcsines, it is computationally expensive to solve for $h$ exactly.

To simplify the angular dependence, we approximate Snell's law with the cubic polynomial that matches the value and derivative of the exact expression at $\pm 90^{\circ}$ :

$$
\gamma_{t}=\frac{3 c}{\pi} \gamma_{i}-\frac{4 c}{\pi^{3}} \gamma_{i}^{3}
$$

where $c=\sin ^{-1}\left(1 / \eta^{\prime}\right)$ (in this expression angles $\gamma$ are measured in radians). The maximum approximation error is less than $0.75^{\circ}$ for $\eta>1.5$.

With this approximation, $\phi$ is a cubic in $\gamma_{i}$ :

$$
\hat{\phi}\left(p, \gamma_{i}\right)=\left(\frac{6 p c}{\pi}-2\right) \gamma_{i}-\frac{8 p c}{\pi^{3}} \gamma_{i}^{3}+p \pi
$$

The roots of this equation yield $\gamma_{i}$ from which $h$ is easily found. For the R and TT cases there will always be exactly one root, whereas the TRT case may have one or three roots.

${ }^{3}$ For the TT and TRT components the shifts depend on the incidence angle, and for all three components the shift depends on $\phi$, but we ignore these effects.

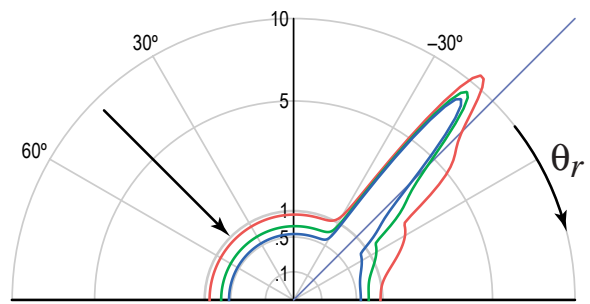

Figure 10: A plot of the shading model, under the same geometric conditions used in Figure 4. The parameters have been adjusted to match the behavior of the blond hair (top center plot in that figure).

\subsubsection{Approximation for TRT}

Our theory, which is based on smooth surfaces, predicts that the caustics in the TRT component produce singularities in $S$ with infinite intensity. Since this is unrealistic, particularly in the presence of surface roughness, we remove the caustic from $N_{T R T}$ and replace it with a smooth lobe centered at the location of the caustic. The width of this lobe simulates the blurring of the caustic due to roughness.

The angle $\phi_{c}$ at which the caustic appears can be computed from Descartes's formula (Equation 4) when it is defined, but for incidence angles past the caustic merge it is undefined. To maintain continuity we continue to insert the caustic at $\phi_{c}=0$ and fade it out smoothly over a short range of incidence angles past the merge. It is simplest to precisely describe this component by giving the procedure used to compute it:

$$
\begin{aligned}
& \text { function } N_{T R T}\left(\theta, \phi ; w_{c}, k_{G}, \Delta \eta^{\prime}, \Delta h_{M}\right) \\
& \text { if }\left(\eta^{\prime}(\theta)<2\right) \\
& \quad \text { Compute } h_{c}, \phi_{c} \text { using } \eta^{\prime}(\theta) \text { in }(4) \\
& \quad \Delta h=\min \left(\Delta h_{M}, 2 \sqrt{2 w_{c} /\left|\frac{d^{2} \phi}{d h^{2}}\left(h_{c}\right)\right|}\right) \\
& \quad t=1 \\
& \text { else } \\
& \quad \phi_{c}=0 \\
& \quad \Delta h=\Delta h_{M} \\
& \quad t=\operatorname{smoothstep}\left(2,2+\Delta \eta^{\prime}, \eta^{\prime}(\theta)\right) \\
& L=N_{p}(2, \phi) \\
& L=L \cdot\left(1-\operatorname{tg}\left(\phi-\phi_{c}, w_{c}\right) / g\left(0, w_{c}\right)\right) \\
& L=L \cdot\left(1-\operatorname{tg}\left(\phi+\phi_{c}, w_{c}\right) / g\left(0, w_{c}\right)\right) \\
& L=L+t k_{G} A(2, \theta, \phi) \Delta h\left(g\left(\phi-\phi_{c}, w_{c}\right)+g\left(\phi+\phi_{c}, w_{c}\right)\right) \\
& \text { return } L
\end{aligned}
$$

In this procedure we roughly approximate the power that is removed from the intensity distribution by using $d^{2} \phi / d h^{2}$ to estimate the size $\Delta h$ of the interval in $h$ that maps within $w_{c}$ of $\phi_{c}$. The limit $\Delta h_{M}$ is necessary because $d^{2} \phi / d h^{2}$ goes to zero at the caustic merge. The function smoothstep $(a, b, x)$ is 1 for $x<a, 0$ for $x>b$, and smooth in between.

The user-adjustable parameters to this function are $w_{c}$, the width of the blur for the caustics, $k_{G}$, a factor to adjust the strength of the glints, $\Delta \eta^{\prime}$, the range of $\eta^{\prime}$ over which to fade out the caustic after the merge, and $\Delta h_{M}$, a limit on the caustic power.

\subsubsection{Approximation for eccentricity}

When the fiber's cross section is elliptical rather than circular, a simple analytical solution for $N$ is not available. However, the appearance of the TRT component is significantly affected by even mild eccentricity: the angle $\phi_{c}$ at which the caustics appear changes by $\pm 100 \%$ over the range from $a=0.85$ to $a=1 / 0.85$. Because eccentricities in this range are very common, it is important to approximate the effect of eccentricity on the TRT component. We do this by using the function $\eta^{*}$ in Equation 9. 
Changing refractive index has effects that are qualitatively similar to changing eccentricity. One case for which it is simple to analyze this relationship in the TRT component is paraxial paths that are symmetric across the major axis. For these paths $\phi_{i}=-\phi_{r}$ and the reflection occurs on the major axis. If $\gamma_{t}^{\prime}$ is the angle of incidence at the internal reflection it can be shown that

$$
\frac{d \phi_{r}}{d \gamma_{t}^{\prime}}=2 a^{2}(\eta-1)-\eta
$$

We can use this first-order approximation to roughly match the behavior of the circular model to the elliptical one by using the index of refraction $\eta^{*}=2 a^{2}(\eta-1)-\eta+2$ in the circular model.

This gives us an approximation for how the TRT component of the scattering function changes with mild eccentricity when $\phi_{h}$ is aligned with one of the principal axes. To incorporate this approximation into the practical model, we simply interpolate sinusoidally to define $\eta^{*}$ for all intermediate values of $\phi_{h}$ :

$$
\begin{aligned}
\eta_{1}^{*} & =2(\eta-1) a^{2}-\eta+2 \\
\eta_{2}^{*} & =2(\eta-1) a^{-2}-\eta+2 \\
\eta^{*}\left(\phi_{h}\right) & =\frac{1}{2}\left(\left(\eta_{1}^{*}+\eta_{2}^{*}\right)+\cos \left(2 \phi_{h}\right)\left(\eta_{1}^{*}-\eta_{2}^{*}\right)\right)
\end{aligned}
$$

As shown in Equation 9, we simulate eccentricity simply by passing the refractive index $\eta^{*}$ to the code that computes $N_{T R T}$ using the formulas derived from the circular case.

\subsection{Summary}

We have now accounted for all the terms that appear in Equation 9. The three longitudinal scattering functions $M_{R}, M_{T T}$, and $M_{T R T}$ are Gaussians. The azimuthal scattering functions $N_{R}$ and $N_{T T}$ are evaluated directly from Equation 8, using Equation 10 to compute $h$. The azimuthal scattering function $N_{T R T}$ is first computed using Equations 8 and 10 with the refractive index $\eta^{*}$, summing over the possibly multiple values of $h$, then modified by the procedure in Section 5.2.2 to smooth out the caustics.

Depending on the type of renderer, it may be helpful in practical use to include a small amount of diffuse reflection. Although our model does not include a diffuse component, one can easily be added by introducing a constant term $M_{D}$ into Equation 9.

All the model's parameters are collected for reference in Table 1.

\section{Results}

\subsection{Validation}

In this section we provide evidence that our assumptions about the structure of hair are valid and that the shading model can qualitatively reproduce the behaviors we have seen in the measurements.

Validating the hair structure model against measurements. The normal-plane measurement in Figure 7, which best illustrates the glints, behaves entirely consistently with the model of a hair as an elliptical cylinder. To show this, we have used a Monte Carlo procedure to compute the scattering due to the TRT mode in a rough elliptical cylinder with eccentricity matching the measured value for this individual. The figure shows the results of this numerical experiment plotted with the results of the physical experiment. The match in both the angular and the intensity variation in the peaks is striking, providing clear evidence both for the explanation of the glints as internal reflection caustics and for the model of the hair's cross section as an ellipse. The real data of course includes some contributions from the other scattering modes, which is noticeable in forward scattering, where the TRT mode does not contribute.

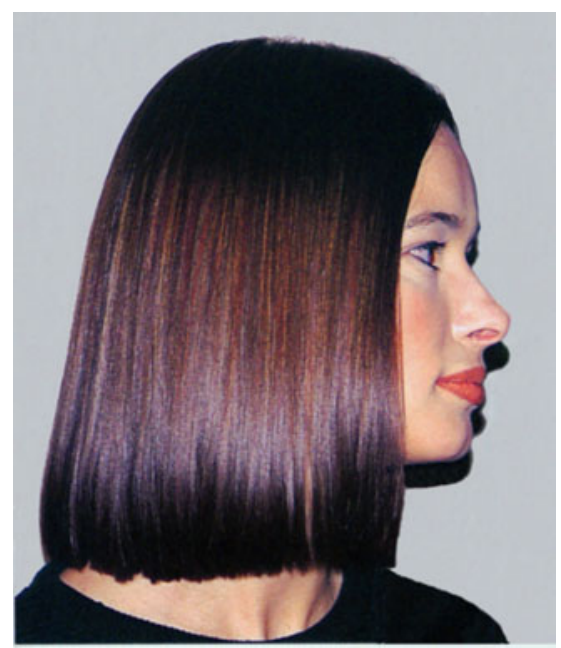

Figure 11: A photograph [Gray 1997] showing brown hair with a prominent secondary highlight caused by a small light source near the camera. Glints help give the hair its distinctive natural texture.

Validating the shading model against measurements. In order to demonstrate the ability of our shading model to qualitatively match the behavior of real hair, we have set the parameters to fit two of the measurements in Section 3. In Figure 10 we show a plot of the model adjusted to behave like the blond hair that was measured in the incidence plane (Figure 4, top center). Note that both the small side lobe and the broader lobe toward grazing are predicted.

The measurement in Figure 6 shows a caustic merge as inclination changes. The shading model is plotted as a dashed line along with the data, with a set of parameters that describes this more mildly eccentric hair. The behavior of two glints merging and disappearing matches qualitatively.

\subsection{Renderings}

We have implemented the shading model described in the previous section and used it to simulate light reflection from procedurally generated assemblies of hair fibers. We have used the commercial "Sasquatch" rendering software (Worley Laboratories, http: //www . worley . com) to model the hair, and each model consisted of 50,000-100,000 spline curves, corresponding to the average number of hairs on a human head. The shading parameters were constant per fiber, but in some cases (noted below) we used a noise function on the scalp to assign a random absorption to each fiber. The azimuthal orientation of the elliptical fibers was random from fiber to fiber but smooth along each fiber. All renderings have $\eta=$ 1.55, and $\Delta h_{M}=0.5$. The parameters $\alpha_{T T}$ and $\alpha_{T R T}$ are defined from $\alpha_{R}$ by the ratios recommended in Table 1 .

We used ray tracing to render the images, accounting for occlusion and shadowing but not multiple scattering between the hairs. We represented the hairs as flat ribbons that always face the ray direction, with a width equal to the hair diameter. This is more efficient than using cylinders, and since our scattering function abstracts away all interactions below the level of strands it is appropriate.

In the following results, computation was dominated by ray intersection so that even though our model is substantially more expensive to compute than Kajiya's very simple model, the increase in rendering time was modest. For instance, switching to the new model increased the rendering time for Figure 12 from 6 minutes to 8 minutes on a two-processor $1 \mathrm{GHz}$ Pentium III system.

The first set of renderings is inspired by the photograph shown in Figure 11. Because the hair is combed into a fairly flat surface, 

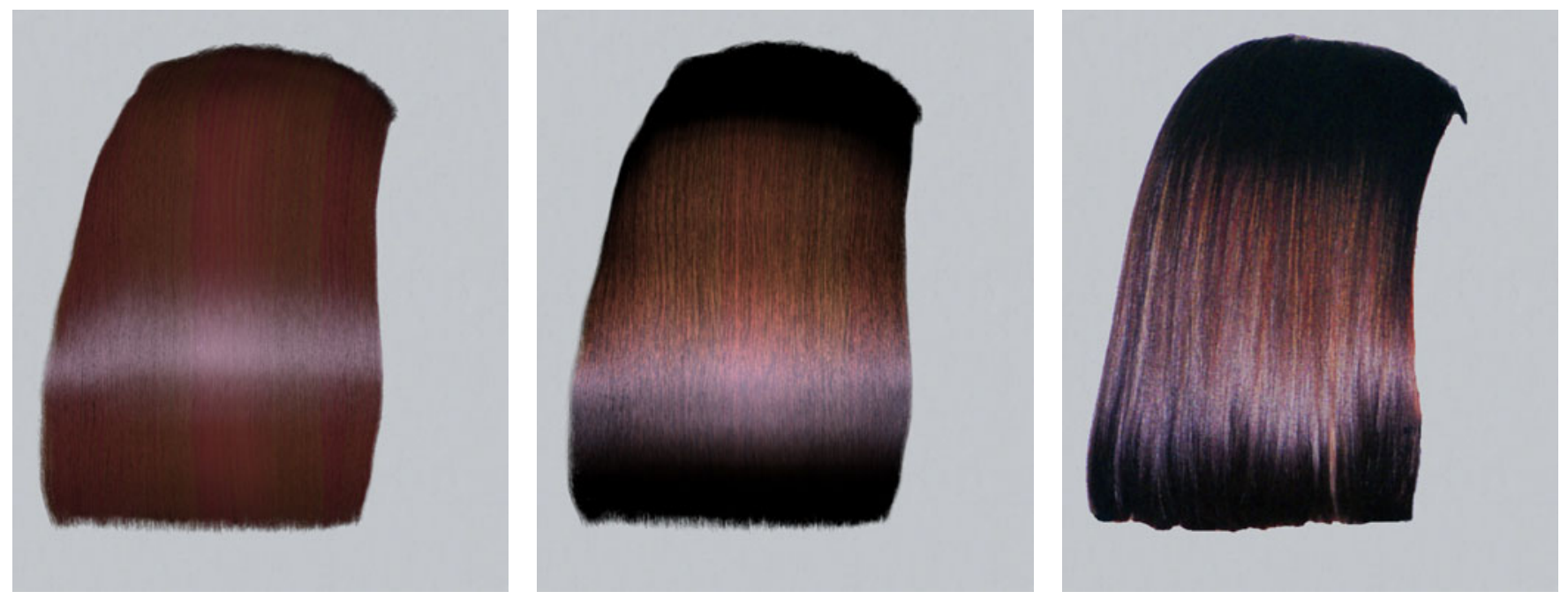

Figure 12: A comparison of Kajiya and Kay's model (left) under a single point source, our proposed model (center) with the same lighting, and the hair from the photograph in Figure 11 (removed from context to simplify the comparison). The Kajiya model's diffuse term results in a flat appearance, while the secondary highlight in our model correctly captures the colored shading of the real hair.

the secondary highlight is quite prominent; there is very little diffuse scattering, as evidenced by the dark appearance at the top and bottom of the hair.

We constructed an idealized geometric model to roughly match the overall shape of the hair, without attempting to reproduce the placement of the fibers exactly. In Figure 12 we show a rendering of this model using settings appropriate for mildly elliptical brown hair. The result of rendering the same model in the same lighting using Kajiya's model is shown for comparison. The diffuse component required to produce an overall brown color does not adequately predict the variation in shading that occurs in the real hair, where the brown color comes from the secondary highlight. The same procedural color variation is present in both images; the additional texture from the proposed model is due to eccentricity. The parameters for this images are $\alpha_{R}=3^{\circ}, \beta_{R}=14^{\circ}, \beta_{T T}=8^{\circ}, \beta_{T R T}=22^{\circ}$, $\sigma_{a}=(0.432,0.612,0.98)-(0.36,0.72,0.94), k_{G}=0.4, w_{c}=1.5^{\circ}$, and $a=0.9$.

To illustrate the components of the model and the effect of eccentricity, Figure 14 shows the two highlights separately and a comparison between circular and elliptical hair with all other parameters held constant. The parameters for these images are $\alpha=3^{\circ}, \beta_{R}=8^{\circ}$, $\beta_{T T}=6^{\circ}, \beta_{T R T}=15^{\circ}, \sigma_{a}=(0.44,0.64,0.9), k_{G}=0.4, w_{c}=1.5^{\circ}$, and $a=1$ and 0.9 .

To further demonstrate the capabilities of the model we include additional results for varying light direction in Figure 15 and for complex illumination (using the method of Agarwal et al. [2003]) in Figure 13.

\section{Conclusions}

In this paper we have looked at light scattering from human hair in reality, in theory, and in rendering practice. Our measurements show that the scattering behavior of individual hair fibers differs significantly from what has previously been assumed in shading models; the theory of scattering from dielectric cylinders explains the sources of these differences; and we present a shading model that captures the relevant visual effects, including those due to eccentricity, in a practical, adjustable model for use in rendering.

The most important implication of this work is that realistic rendering of hair needs to account for the multiple modes of scattering that occur in cylinders. The standard assumption that the color comes primarily from diffuse scattering is not supported by the evidence in the case of fibers, and our images show that this assumption limits the quality of results from current hair shading models.

The shading model we have introduced could be made more accurate in future work. Two avenues for improvement are eccentricity and surface roughness. It would be useful to handle more extreme deviations from circularity, in order to allow us to accurately model more types of hair. Also, the current model approximates the effects of eccentricity on the ray density factor but not the Fresnel factor, which limits the quantitative accuracy of the energy distribution. Our model accounts for the effects of surface roughness and the scale angle in the simplest way that is consistent with the appearance phenomena. The true effects of these surface features are complicated and dependent on the scattering geometry. A more exact model of these effects could lead to a scattering model that is more accurate for grazing angles.

Our analysis applies to many kinds of transparent fibers, so it has implications for rendering animal hair, synthetic hair or fur, and all kinds of cloth.

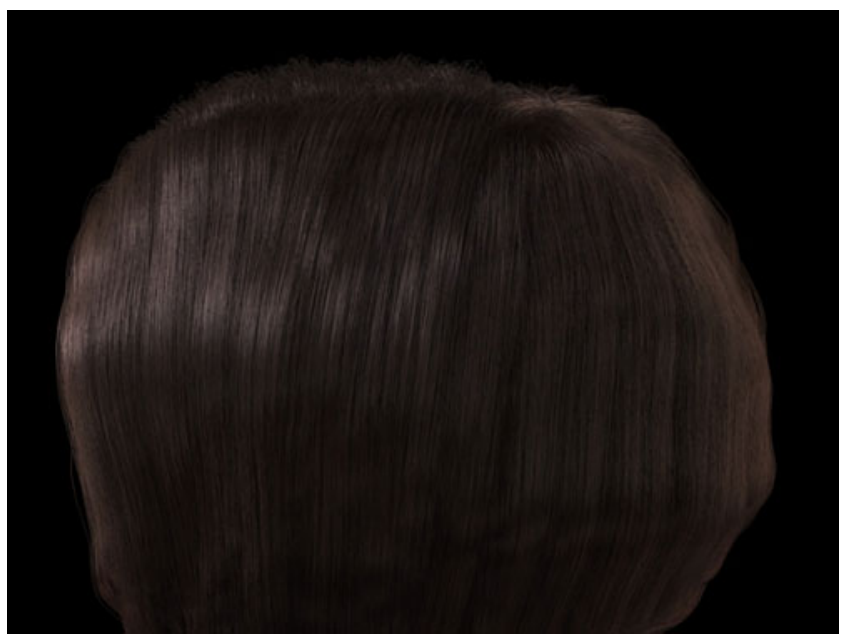

Figure 13: A hair model (62K fibers) illuminated by a complex environment map. 


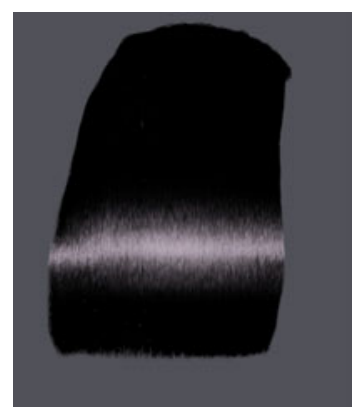

(a)

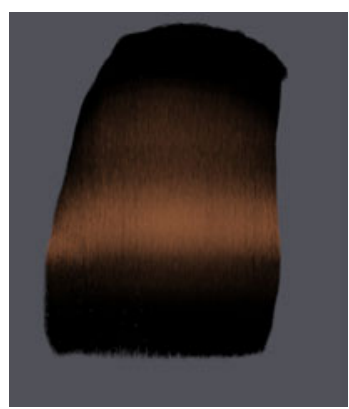

(b)

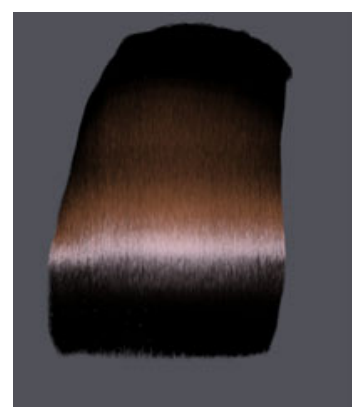

(c)

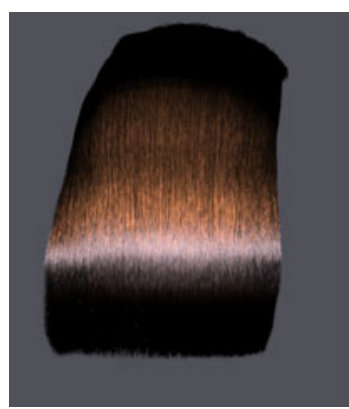

(d)

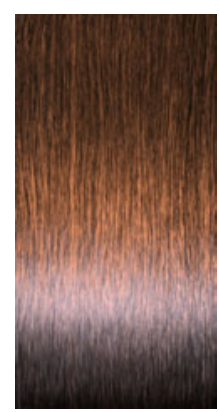

(e)

Figure 14: $(\mathrm{a}-\mathrm{c})$ The components of our model for circular hair $(a=1)$ : (a) the R component (primary highlight) alone; (b) the TRT component (secondary highlight) alone; (c) the full model. (d) The full model for $a=0.9$, showing texture due to glints; (e) an enlargement of $(\mathrm{d})$.
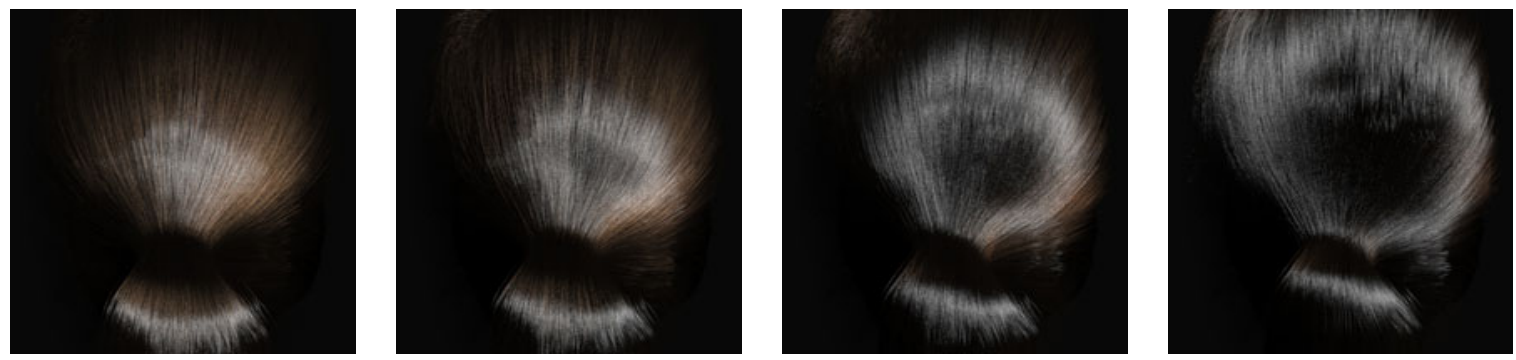

Figure 15: A hair model under different illumination angles. As the relative strengths of the primary and secondary highlights change, they alter the color, brightness, and sheen of the hair.

\section{Acknowledgements}

The authors would like to thank Heidi Marschner, Joyce Ma, and James Davis for donating the hairs we measured, François Guimbretière for the presentation idea for Figure 7, and Dr. John Gray for the use of the photograph in Figure 11. This research was supported by an NSF ITR grant (IIS-0085864), and the third author was supported by a 3Com Corporation Stanford Graduate Fellowship and an NSF Graduate Fellowship. The scattering measurements were performed on the Stanford Spherical Gantry, which was designed by Marc Levoy, Brian Curless, and Szymon Rusinkiewicz and constructed using funds from Interval Research.

\section{References}

AdleR, C. L., Lock, J. A., AND Stone, B. R. 1998. Rainbow scattering by a cylinder with a nearly elliptical cross section. Applied Optics 37, 9, $1540-1550$.

Agarwal, S., Ramamoorthi, R., Belongie, S., And Jensen, H. W. 2003. Structured importance sampling of environment maps. ACM Transactions on Graphics 22, 3.

Bustard, H., AND SMITH, R. 1991. Investigation into the scattering of light by human hair. Applied Optics 24, 30, 3485-3491.

Chang, J., Jin, J., AND YU, Y. 2002. A practical model for hair mutual interactions. In Proceedings of the ACM SIGGRAPH Symposium on Computer Animation, ACM Press, 73-80.

Chen, L.-H., Saeyor, S., Dohi, H., And Ishizuka, M. 1999. A system of 3D hair style synthesis based on the wisp model. The Visual Computer 15, 4, 159-170.

Goldman, D. B. 1997. Fake fur rendering. In Proceedings of SIGGRAPH 1997, ACM Press, 127-134.

GRAY, J. 1997. World of Hair. Hairdressing Training Board.
Hadap, S., AND Magnenat-Thalmann, N. 2000. Interactive hair styler based on fluid flow. In Computer Animation and Simulation 2000, Eurographics, 87-99.

Humphreys, W. J. 1964. Physics of the Air. Dover, London.

KAJIYA, J. T., AND KAY, T. L. 1989. Rendering fur with three dimensional textures. In Proceedings of SIGGRAPH 1989, 271-280.

KAJIYA, J. T. 1985. Anisotropic reflection models. In Proceedings of SIGGRAPH 85, ACM Press, 15-21.

Kim, T.-Y., AND Neumann, U. 2000. A thin shell volume for modeling human hair. In Computer Animation 2000, IEEE CS Press, 104-111.

Kim, T.-Y., And Neumann, U. 2001. Opacity shadow maps. In Rendering Techniques 2001: Proceedings of Eurographics Rendering Workshop, Springer Verlag Wien, 177-182.

KIM, T.-Y., AND NEUMANN, U. 2002. Interactive multiresolution hair modeling and editing. ACM Transactions on Graphics 21, 3, 620-629.

KIM, T.-Y. 2002. Modeling, Rendering, and Animating Human Hair. $\mathrm{PhD}$ thesis, University of Southern California.

KonG, W., AND NAKAJima, M. 2000. Hair rendering by jittering and pseudo shadow. In Computer Graphics International 2000, 287-294.

Lokovic, T., AND Veach, E. 2000. Deep shadow maps. In Proceedings of SIGGRAPH 2000, ACM Press, 385-392.

Magnenat-Thalmann, N., Hadap, S., And Kalra, P. 2002. State of the art in hair simulation. In International Workshop on Human Modeling and Animation, Korea Computer Graphics Society, 3-9.

MARCUSE, D. 1974. Light scattering from elliptical fibers. Applied Optics 13, 1903-1905.

Mount, C. M., Thiessen, D. B., And Marston, P. L. 1998. Scattering observations for tilted transparent fibers. Applied Optics 37, 9, 15341539.

POUlin, P., AND FouRnier, A. 1990. A model for anisotropic reflection. In Proceedings of SIGGRAPH 90, 273-282. 

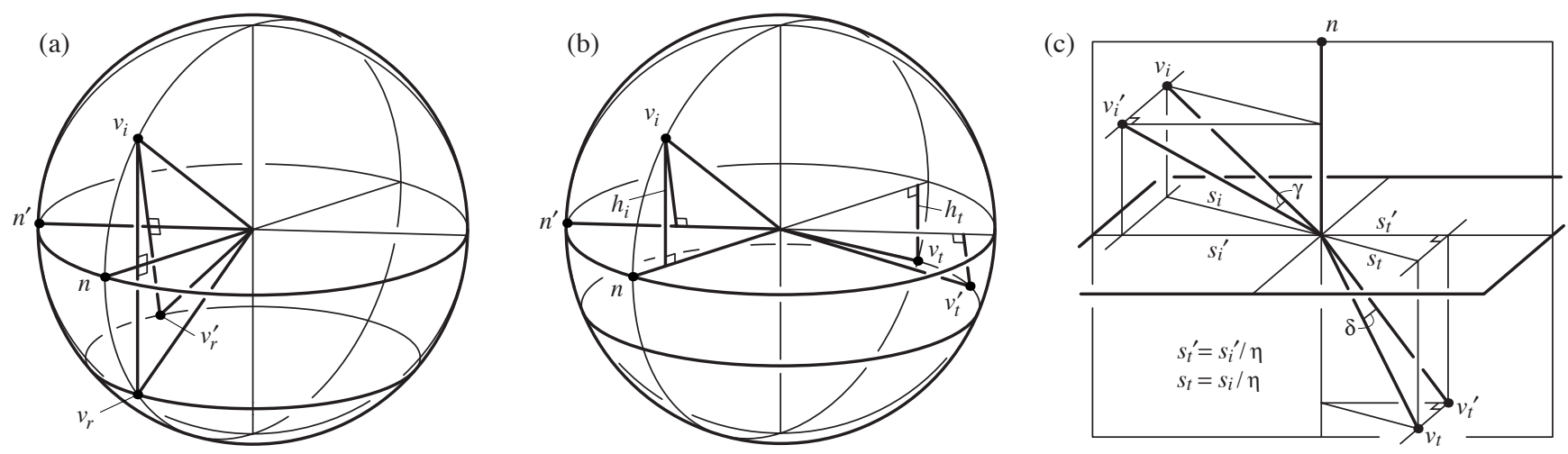

Figure 16: Reflected (a) and refracted (b) vectors from a cylinder lie in a cone; derivation of Bravais's law.

RobBins, C. R. 1994. Chemical and Physical Behavior of Human Hair, third ed. Springer-Verlag, New York.

Sourin, A., PASKo, A., AND SAVCHENKo, V. 1996. Using real functions with application to hair modelling. Computers \& Graphics 20, 1, 11-19.

Stamm, R. F., GARCiA, M. L., AND Fuchs, J. J. 1977. The optical properties of human hair I. fundamental considerations and goniophotometer curves. J. Soc. Cosmet. Chem., 28, 571-600.

TRICKER, R. A. R. 1970. Introduction to Meteorological Optics. Mills \& Boon, London.

WATANABE, Y., AND SUENAGA, Y. 1992. A trigonal prism-based method for hair image generation. IEEE Computer Graphics \& Applications 12, $1,47-53$.

\section{A Loci of reflections}

In this appendix, the geometry of reflection and refraction from a cylindrical fiber is analyzed. The set of normal vectors to a cylindrical fiber lie in a plane perpendicular to the axis of the fiber; this plane is called the normal plane. The geometry is most easily visualized using a unit sphere; each point on the unit sphere represents a different direction. On this sphere, the axis of the fiber is at the north pole and the set of normal vectors is contained in the equator (see Figure 16a).

To analyze reflection for a given incident direction $v_{i}$, consider a particular surface normal $n$ and the corresponding reflection vector $v_{r}$. The law of reflection states that $v_{i}, n$, and $v_{r}$ are coplanar and $n \cdot v_{i}=n \cdot v_{r}$. By finding similar triangles, the reflection rule also forces the distances of $v_{i}$ and $v_{r}$ from the equator to be equal. This equidistance condition must hold for any normal vector. Thus, the set of reflection vectors formed for a given incident direction must lie on a horizontal circle at constant height that is at the same distance from the equator as $v_{i}$. This is a formal proof of the wellknown result that, in $3 \mathrm{D}$, the set of reflections from a cylinder form a cone.

Figure 16b is analogous to the previous figure, but shows the refracted directions instead. The rule for refraction (Snell's law) is similar to reflection. First, $v_{i}, n$, and $v_{t}$ are coplanar. Second, Snell's Law says that $\eta \sin \theta_{t}=\sin \theta_{i}$, and using similar triangles, this law implies that the perpendicular distances to the equator, $h_{i}$ and $h_{t}$, also have the ratio $\eta$. This means the transmitted vectors all lie on a circle that is a factor of $\eta$ closer to the equator than $v_{i}$.

By the same argument, the rays that refract again on the way out of the cylinder will obey the equidistance condition. Furthermore, internal reflections are no different than external reflections. The conclusion is that all rays that exit a cylindrical fiber must lie in the same cone as the reflected rays.

\section{B The Bravais index}

A cylindrical fiber is formed by sweeping a cross section along an axis. Bravais has shown that the optics of a 3D cylindrical fiber may be reduced to the $2 \mathrm{D}$ analysis of the optics of its cross section. The incident direction is first projected into the cross-section plane and then reflected and refracted in 2D. However, to model the correct physics in $2 \mathrm{D}$, the index of refraction must be changed as a function of the angle of incidence. Bravais's law is often used to analyze refraction through crystals (as in ice haloes [Tricker 1970]).

Figure $16 \mathrm{c}$ shows the geometry of the refraction across a horizontal surface. The direction $v_{i}$ is refracted in the direction $v_{t}$. To reduce this to a $2 \mathrm{D}$ problem, the vectors $v_{i}$ and $v_{t}$ are projected onto the vertical plane containing the normal. The projected vectors are labeled $v_{i}^{\prime}$ and $v_{t}^{\prime}$. The goal is to find an effective index of refraction, $\eta^{\prime}$, such that if $v_{i}^{\prime}$ is refracted at the boundary it will yield $v_{t}^{\prime}$. First note that Snell's Law states that $\sin \theta_{i}=\eta \sin \theta_{t}$. That is, the length of the projection of $v_{i}$ onto the horizontal plane is $\eta$ times the length of the projection of $v_{t}$. Since $v_{i}$ and $v_{t}$ lie in a plane, the two triangles shown in the horizontal plane are similar. The length of each edge of the left (incident-side) triangle is $\eta$ times the length of the corresponding edge in the right (transmitted-side) triangle. Second, an equivalent Snell's Law is obeyed in the vertical normal plane. Here, $\sin \theta_{i}^{\prime}=\eta^{\prime} \sin \theta_{t}^{\prime}$. But $\sin \theta_{i}^{\prime}=\sin \theta_{i} \cos \gamma$, where $\gamma$ is the angle between the incident vector and its projection on the normal plane. Similarly, $\sin \theta_{t}^{\prime}=\sin \theta_{t} \cos \delta$, where $\delta$ is the angle between the transmitted direction and its projection on the normal plane. Substituting, yields $\sin \theta_{i} \cos \gamma=\eta^{\prime} \sin \theta_{t} \cos \delta$, and substituting Snell's Law into this equation yields $\eta \cos \gamma=\eta^{\prime} \cos \delta$, Equivalently,

$$
\eta^{\prime}(\gamma)=\sqrt{\eta^{2}-\sin ^{2} \gamma} / \cos \gamma
$$

The implication of this is that for an incident ray that makes an angle $\gamma$ with the projection plane, we can compute the projection of the refracted direction from the projection of the incident direction using the usual Snell's law but substituting the effective index of refraction $\eta^{\prime}$.

The Fresnel attenuation at the interface may be computed in a similar way. From Fresnel's formulas, it is straightforward to verify that the factor for oblique incidence may be computed from the projected angles by using $\eta^{\prime}$ for the perpendicular component of the reflectance and a second index,

$$
\eta^{\prime \prime}(\gamma)=\eta^{2} \cos \gamma / \sqrt{\eta^{2}-\sin ^{2} \gamma}
$$

for the parallel component. We will use the notation $F\left(\eta^{\prime}, \eta^{\prime \prime}, \gamma\right)$ for this slightly generalized Fresnel function. 